\title{
Physiotherapy Management of Faecal Impaction: A case report
}

\author{
Kareem, R.O., ${ }^{1}$ Ayanniyi, O., ${ }^{1}$ Ajiboye, O.A., ${ }^{2}$ Hamzat, T.K. ${ }^{1}$ \\ ${ }^{1}$ Department of Physiotherapy, College of Medicine, University of Ibadan, Ibadan \\ ${ }^{2}$ Department of Physiotherapy, Lagos University Teaching Hospital, Idi-Araba, Lagos \\ Correspondence \\ R.O. Kareem, Department of Physiotherapy, College of Medicine, University of Ibadan, Ibadan \\ Email:agbeloba007@yahoo.com
}

\begin{abstract}
SUMMARY
This case report describes how a physiotherapy modality was used to resolve faecal impaction. A 38-yearold man developed faecal impaction after 8 weeks of intensive medical care. A physiotherapist was invited to apply a suitable modality that could bring relief to the patient. The pre-treatment measurement of the distended abdomen was $102 \mathrm{~cm}$. Modified passive mobilization of the lower limbs towards the abdominal surface was carried out. After 6 sessions, the faecal impaction was resolved. The post-treatment measurement of the abdomen was $86.5 \mathrm{~cm}$.

This case demonstrates the important role of a physiotherapy modality in the management of faecal impaction. It is hoped that this study will stimulate the interest of physiotherapists in the management of this condition.
\end{abstract}

KEY WORDS: faecal impaction, megacolon, modified passive mobilization, abdominal distention

\section{INTRODUCTION}

Faecal impaction is a large mass of dry, hard stool that accumulates in the rectum due to unresolved constipation (Creason and Sparks, 2000). The dryness of the mass makes it very difficult to excrete. Watery stool from a higher level in the bowel may move around the mass and leak out, causing soiling. The longer the patient is unable to expel the stool, it further accumulates into a larger, harder mass that is difficult to pass by normal defaecation. This accumulated stool can extend up into the sigmoid colon (the loop of the larger bowel above the rectum) leading to serious discomfort. This accumulation can also lead to generalized abdominal distention.

Faecal impaction is more common in elderly people with limited ability to move (Saddler, 2005). Among the causes are medications like antacids, which have aluminium as an ingredient; calcium and iron supplements; a category of antihypertensive drugs known as calcium-channel blockers; psychotropic medications, such as antidepressants and tranquillizers, and drugs to reduce spasms, such as those used in treating Parkinson's disease. Other causes of faecal impaction are poor bowel habits, inadequate fluid intake, a diet that is lacking in fibres and rectal disorders (rectocele).

Untreated faecal impaction can also become a serious health problem resulting in megacolon (a massively swollen colon, usually associated with severe and chronic constipation that can exist as a congenital condition or develop later in life), which usually requires surgical correction. It is possible for rectal tissues to become damaged, ulcerated and even necrotic. The most serious potential complication is the total obstruction of the lower bowel, which can be potentially fatal.

\section{CASE REPORT}

The patient was a 38-year-old man under intensive care at the Medical Intensive Care Unit of Lagos University Teaching Hospital, Idi-Araba, Lagos. He was referred for physiotherapy management with a diagnosis of respiratory failure, aspiration pneumonia and transverse myelitis.

\footnotetext{
This case report was presented at the $46^{\text {th }}$ Annual Scientific Conference of the Nigeria Society of Physiotherapy, November 2006.
} 


\section{Physiotherapy Management}

After 8 weeks under intensive care, the patient developed faecal impaction. He was given stool softeners, adequate fluid intake and a diet rich in fibre, but the faecal impaction did not resolve. Digital removal of the impacted stool was carried out by the nurses, but it did not completely resolve the faecal impaction. A physiotherapist was then invited by the medical team to apply a suitable modality that could bring relief to the patient.

\section{Initial Physiotherapy Assessment}

The initial physiotherapy assessment found that:

- the patient was unconscious with a Glasgow Coma Scale (GSC) of $5 / 15$

- the patient was placed on a respirator and a cardiac monitor

The initial aims of physiotherapy management were:

- to ensure and maintain a patent airway

- to maintain the physiological properties of the muscles and joints

- to prevent/delay the onset of the other complications associated with immobility

\section{Physiotherapy Re-assessment}

A re-assessment of the patient was carried out six months after the initial assessment. On examination:

- the patient was quadriplegic

- the patient had a swollen and shining abdomen

- there were signs of pain distress

- there was a hard mass of stool in the anal orifice

- the abdominal circumference, measured with a tape rule, was $102 \mathrm{~cm}$

Landmarks: $15 \mathrm{~cm}$ below the site of the xiphoid process along a straight line between the site of the xiphoid process and the site of pubic tubercle.

\section{Treatment Procedure}

After wearing a pair of latex gloves and face masks, the 2 physiotherapists positioned the patient in a supine position and tilted him as gently as possible to about $45^{\circ}$, using a pillow. A water proof pad was placed under the patient's buttocks and a nurse was in attendance with a bedpan to hold any removed stool.
The physiotherapists, one on each side of the patient, used one hand to cradle the patient's heel and put the other hand in the popliteal fossa and lifted the lower extremities simultaneously, while allowing the hip and knee joints to flex. The hip joints were fully flexed and adducted over the abdominal surface with an over pressure sustained for 7 seconds as gently as possible, followed by an immediate rest period of 60 seconds. This manoeuvre was aimed at simulating the squatting position in the patient. Immediately the manoeuvre started, the impacted stool was seen being dislodged downward towards the waterproof pad. The procedure was repeated 5 times. During the procedure, eye contact was maintained with the patient to ensure that there were no adverse effects, such as diaphoresis and changes in pulse rate.

\section{Outcome of Physiotherapy Management}

After 6 treatment sessions, each comprising 5 repetitions of passive flexion of the hip and knee joints with the thigh pressing against the abdominal surface, the faecal impaction was completely resolved. Each treatment session lasted ten minutes. The 6 sessions were spread over a period of 4 days. At the end of the period, the swollen and shining abdomen was reduced with a posttreatment abdominal circumference of $86.5 \mathrm{~cm}$, compared to the pre-treatment measurement of $102 \mathrm{~cm}$. In addition, the patient was followed up for a 6-week period to ensure there was no recurrence of faecal impaction. During the follow-up period, the abdominal circumference further dropped to $85.5 \mathrm{~cm}$.

\section{DISCUSSION}

Traditionally, the treatment of faecal impaction involved the digital breaking and removal of the impacted stool, as well as measures to prevent constipation and further development of impaction (Kyle et al, 2005). This procedure can potentially damage the mucous membrane lining of the rectum and cause stimulation of the vagus nerve which has endings in the rectum; this could result in heart irregularities.

The physiotherapy management of the faecal impaction in this case report demonstrates the effectiveness of the combination of gravity-assisted position and a passive flexion of the hip and knee joints, with the thigh pressing against the abdominal surface. This procedure may have resulted in increased intraabdominal and intra-rectal pressure, which may have been accompanied by the reflex dilatation of the anal 
orifice that gently dislodged the impacted stool with relatively low risk and no adverse effects.

Although this method has only been used once, it is a procedure which can be employed by physiotherapists as one of the modalities for managing faecal impaction. Further studies could also be carried out to evaluate its potential uses.

\section{References}

Creason, N. and Sparks, D. (2000) Faecal impaction: A review. Nursing Diagnosis 11, pp.15-23.

Kyle, G., Prynn, P. and Oliver H. (2005) A procedure for the digital removal of faeces. Nursing Standard 1, pp.33-39.

Saddler, D. (2005) A literature review of faecal impaction. Gastroenterology Nursing 28, pp.49-50. 